\title{
Estimates of the first eigenvalue of minimal hypersurfaces of $\mathbb{S}^{n+1}$
}

\author{
Abdênago Barros G. Pacelli Bessa*
}

September 26, 2018

\begin{abstract}
We consider a solution $f$ of a certain Dirichlet problem on a domain in $\mathbb{S}^{n+1}$ whose boundary is a minimal hypersurface and we prove a Poincaré type inequality for $f$. Moreover we have an estimate for the first nonzero eigenvalue for the closed eigenvalue problem on the boundary.
\end{abstract}

\section{Introduction}

In this note we will let $M^{n}$ be an embedded compact orientable minimal hypersurface of $\mathbb{S}^{n+1}$. Yau conjectures that the first nonzero eigenvalue $\lambda_{1}(M)$ for the closed eigenvalue problem $\triangle_{M} u+\lambda u=0$ on $M$ was equal to $n$. Observe that $M$ divided $\mathbb{S}^{n+1}$ into two connected components $\Omega_{1}$ and $\Omega_{2}$ such that $\partial \Omega_{1}=\partial \Omega_{2}=M$. Choi-Wang [1] with a clever idea, applied Reilly formula to the solution of the following Dirichlet problem,

$$
\left\{\begin{array}{l}
\bar{\triangle}=0 \text { on } \Omega_{1} \\
f=\varphi \text { on } M,
\end{array}\right.
$$

where $\varphi$ is the first eigenfunction for the closed eigenvalue problem on $M$ to prove that $\lambda_{1}(M)>n / 2$. We improve (conceptually) Choi-Wang's estimates in terms of the soluition $f$ of the problem (11), (see Corollary 1.2. inequality 3) with possibility to set up Yau's conjecture provided one proves equality in 5 . The symbols $\bar{\triangle}$ and $\bar{\nabla}$ will be respectively the Laplacian and gradient of the metric of $\mathbb{S}^{n+1}$ on $\Omega_{1}$ while $\triangle$ and $\nabla$ will be the Laplacian and gradient oh the induced metric on $M$.

*bessa@mat.ufc.br, abbarros@mat.ufc.br 
thm1 Theorem 1.1 Let $f$ be the solution of the Dirichlet problem (11). Then

$$
\begin{aligned}
p(t) & =\left(2 \lambda_{1}(M)-n\right)\|\bar{\nabla} f\|^{2} \cdot t^{2} \\
& +2 \lambda_{1}(M)\|f\|^{2} \cdot t+\frac{n}{n+1}\|f\|^{2} \geq 0, \quad \forall t \in \mathbb{R}
\end{aligned}
$$

where \|\| denotes the $L^{2}$ norm on $\Omega_{1}$.

corollary1 Corollary 1.2 Let $M$ be an orientable embedded minimal hypersurface of $\mathbb{S}^{n+1}$ and $\lambda_{1}(M)$ its first non-zero eigenvalue of the Laplacian for closed eigenvalue problem on $M$. Consider the problem (1) and $f$ its solution. Then

$$
\lambda_{1}(M) \geq \frac{n}{2}+\frac{n}{2} \rho(f)
$$

where

$$
\rho(f)=\frac{2\|\bar{\nabla} f\|^{2}-(n+1)\|f\|^{2}-2\|\bar{\nabla} f\|^{2} \sqrt{1-(n+1) \frac{\|f\|^{2}}{\|\bar{\nabla} f\|^{2}}}}{(n+1)\|f\|^{2}}
$$

Observe that $0<\rho(f) \leq 1$ and $\rho(f)=1$ iff $\|\bar{\nabla} f\|^{2}=(n+1)\|f\|^{2}$. Although the function $f$ does not belong to $H_{0}^{1}\left(\Omega_{1}\right)$ we have the following Poincaré type inequalities.

Corollary 1.3 Let $f$ be the solution of the Dirichlet problem (1). Then $f$ satisfies the following inequalities:

$$
\begin{gathered}
\|\bar{\nabla} f\|^{2} \geq(n+1)\|f\|^{2} \\
\left\|\bar{D}^{2} f\right\|^{2}>\frac{n(n+1)}{4}\|f\|^{2},
\end{gathered}
$$

where $\bar{D}^{2} f$ is the Hessian of $f$.

\section{Prof of the Results}

Let $\Omega$ be a Riemannian manifold of dimension $n$ with smooth boundary $\partial \Omega$ and let $f$ be a function on $\Omega$ which is smooth up to the boundary $\partial \Omega$. We let $\varphi=f \mid \partial \Omega$ and $u=\partial f / \partial \nu$ the normal outward derivative of $f$. For $X, Y \in T \Omega,\left(\bar{D}^{2} f\right)(X, Y)$ denotes the Hessian tensor. Let $B(v, w)$ be the second fundamental form of $\partial \Omega$ relative to $\Omega$. Here $v, w$ 
are tangent to $\partial \Omega, H$ is the mean curvature of $\partial \Omega$ and ric is the Ricci curvature of $\Omega$. The following identity is known as the Reilly formula.

$$
\begin{aligned}
\int_{\Omega}(\bar{\triangle} f)^{2}= & \int_{\Omega}\left|\bar{D}^{2} f\right|+\int_{\Omega} \operatorname{Ric}(\bar{\nabla} f, \bar{\nabla} f)+\int_{\partial \Omega} 2 u \Delta \varphi \\
& +\int_{\partial \Omega} B(\nabla \varphi, \nabla \varphi)+\int_{\partial \Omega} n H u^{2}
\end{aligned}
$$

Now we can show the proof of Theorem (1.1). If $t=0$ we are done. Now, for $t \neq 0$ we consider the following Dirichlet problem

$$
\left\{\begin{array}{l}
\bar{\triangle}=f \text { on } \Omega_{1} \\
g=t \varphi \text { on } M
\end{array}\right.
$$

Applying the Green formula we obtain

$$
\left\{\begin{array}{l}
\int_{M} \varphi \frac{\partial f}{\partial \nu}=\int_{\Omega_{1}}|\bar{\nabla} f|^{2} \\
t \int_{M} \varphi \frac{\partial f}{\partial \nu}=\int_{\Omega_{1}}\langle\bar{\nabla} f, \bar{\nabla} g\rangle \\
\int_{M} \varphi \frac{\partial g}{\partial \nu}=\int_{\Omega_{1}} f^{2}+\int_{\Omega_{1}}\langle\bar{\nabla} f, \bar{\nabla} g\rangle
\end{array}\right.
$$

From(9) we get

$$
\int_{\Omega_{1}}\langle\bar{\nabla} f, \bar{\nabla} g\rangle=t \int_{\Omega_{1}}|\bar{\nabla} f|^{2}
$$

eq10

and by Cauchy-Schwarz inequality we get

$$
\int_{\Omega_{1}}|\bar{\nabla} g|^{2} \geq t^{2} \int_{\Omega_{1}}|\bar{\nabla} f|^{2}
$$

from the third equation in (9) and (10) we have that

$$
t \int_{M} \varphi \frac{\partial g}{\partial \nu}=t \int_{\Omega_{1}} f^{2}+t^{2} \int_{\Omega_{1}}|\bar{\nabla} f|^{2}
$$

Applying Reilly formula to $g$, using the fact that $\left|\bar{D}^{2} g\right|^{2} \geq \frac{1}{n+1}(\bar{\triangle} g)^{2}$ and the assumption that $\int_{M} B(\nabla \varphi, \nabla \varphi) \geq 0$ we have

$$
\frac{n}{n+1} \int_{\Omega_{1}}(\bar{\triangle} g)^{2} \geq n \int_{\Omega_{1}}|\bar{\nabla} g|^{2}+2 \int_{M} \frac{\partial g}{\partial \nu}(\triangle t \varphi)
$$


On the other hand, taking in account (11), (12) and $\bar{\triangle} g=f$ we have that (13) implies that

$$
\frac{n}{n+1} \int_{\Omega_{1}} f^{2} \geq n t^{2} \int_{\Omega_{1}}|\bar{\nabla} f|^{2}-2 \lambda_{1}(M)\left[t \int_{\Omega_{1}} f^{2}+t^{2} \int_{\Omega_{1}}|\bar{\nabla} f|^{2}\right]
$$

Therefore we have

$$
p(t)=\left(2 \lambda_{1}(M)-n\right)\|\bar{\nabla} f\|^{2} t^{2}+2 \lambda_{1}(M)\|f\|^{2} t+\frac{n}{n+1}\|f\|^{2} \geq 0 .
$$

This finishes the proof of Theorem (1.1). The discriminant of $p$ is nonpositive. This can be read as follows

$$
\left(2 \lambda_{1}(M)-n\right) \geq \frac{n+1}{n} \lambda_{1}(M)^{2} \frac{\|f\|^{2}}{\|\bar{\nabla} f\|^{2}}
$$

From (16) and (3) we have the following poincaré inequality for $f$,

$$
\|\bar{\nabla} f\|^{2} \geq(n+1)\|f\|^{2}
$$

In the proof of Theorem (1.1) we did not count on with an extra term $\int_{M} B(\nabla t \varphi, \nabla t \varphi) \geq 0$ on the right side of (13). Taking it in account we have in fact that $p(t) \geq \int_{M} B(\nabla \varphi, \nabla \varphi) \cdot t^{2}$. From that we can conclude that

$$
\left(2 \lambda_{1}(M)-n\right)\|\bar{\nabla} f\|^{2}-\int_{M} B(\nabla \varphi, \nabla \varphi) \geq \frac{n+1}{n} \lambda_{1}(M)^{2} \frac{\|f\|^{2}}{\|\bar{\nabla} f\|^{2}}
$$

On the other hand, Reilly formula also gives

$$
\left(2 \lambda_{1}(M)-n\right)\|\bar{\nabla} f\|^{2}=\left\|\bar{D}^{2} f\right\|^{2}+\int_{M} B(\nabla \varphi, \nabla \varphi) .
$$

Therefore we obtain

$$
\left\|\bar{D}^{2} f\right\|^{2} \geq \frac{n+1}{n} \lambda_{1}(M)^{2}\|f\|^{2}>\frac{n(n+1)}{4}\|f\|^{2},
$$

since $\lambda_{1}(M)>n / 2$.

\section{References}

[1] Choi, H. I., Wang, A. N. : A first eingenvalue estimate for minimal hypersurfaces. J. Diff. Geom. 18, 559-562 (1983). 